\title{
Composição bromatológica da silagem de quatro genótipos de girassol, ensilados em cinco diferentes idades de corte
}

[Bromatological composition of silage of four sunflower genotypes ensiled at different harvesting ages]

\author{
B.P.S. Souza ${ }^{1}$, S.G. Coelho ${ }^{2}$, L.C. Gonçalves ${ }^{2}$, F.A.P. Vieira ${ }^{1}$, A.L.C.C. Borges ${ }^{2}$, N.M. Rodriguez ${ }^{2}$, \\ J.A.S. Rodrigues ${ }^{3}$, I. Borges ${ }^{2}$, E.S. Saliba ${ }^{2}$ \\ ${ }^{1}$ Pós-graduando - Escola de Veterinária da UFMG \\ ${ }^{2}$ Departamento de Zootecnia - Escola de Veterinária da UFMG \\ Caixa Postal 567 \\ 30123-970 - Belo Horizonte, MG \\ ${ }^{3}$ Embrapa Milho e Sorgo - Sete Lagoas, MG
}

\begin{abstract}
RESUMO
Determinaram-se a composição bromatológica do material original e das silagens de quatro genótipos de girassol (M742, MG4, PM92007 e VDH483) utilizados para produção de grãos, ensilados em diferentes idades de corte após o plantio e aos 90, 97, 104, 111, e 118 dias, e o melhor momento de colheita desses cultivares para ensilagem. Os teores de matéria seca variaram entre as idades e cultivares, sendo observados valores de $12,7 \%$ a $42,2 \%$ no material original e $11,1 \%$ e $40,2 \%$ na silagem. Os valores de proteína bruta variaram de $8,3 \%$ a $12,6 \%$ no material original e de $9,4 \%$ a $12,9 \%$ na silagem. Foi observada diminuição $(\mathrm{P}<0,05)$ nos teores de carboidratos com a ensilagem. Os teores de extrato etéreo do material original e das silagens variaram de 2,2 a $13,3 \%$ no material original e de 3,0 a $17,9 \%$ na ensilagem. Houve diminuição dos teores de hemicelulose com a ensilagem, sugerindo que essa fração foi utilizada com substrato adicional para fermentação. Os teores de ácido lático diminuíram com o estágio de maturação da planta. Houve diminuição nos valores de digestibilidade in vitro da matéria seca com o avanço do estádio de maturação da planta. O melhor momento de colheita para os genótipos M742, MG4 e VDH483 foi aos 104 dias de idade e para o genótipo PM92007, aos 111dias.
\end{abstract}

Palavras-chave: girassol, cultivar, digestibilidade, matéria seca, in vitro, idade de corte, $\mathrm{pH}$

\begin{abstract}
The bromatological composition of four sunflower genotypes (M742, MG4, PM92007 and VDH483) used for producing seed, ensiled at different harvest ages (90, 97, 104, 111 and 118 days) after sowing, was used to determine the best time to ensile. Differences among ages and cultivars were found for dry matter of the genotypes. The crude protein values varied from 8.3 to $12.6 \%$ for the original sample and from 9.4 to $12.9 \%$ for the silage. A decrease $(P<0.05)$ in the soluble carbohydrate values for the ensiled genotypes was observed. The ether extract values varied from 2.2 to $13.3 \%$ for the original sample and from 3.0 to $17.9 \%$ for the silage. The values of hemicellulose and lignin decreased with the ensiling process, suggesting the fibrous fractions contributed, as additional substrate source, for fermentation. The lactic acid values decreased with the plant maturity stage. The dry matter in vitro digestibility decreased with the plant maturity. The best time to ensile, based on the quality of the sunflower genotype M742, MG4 and VDH483 silage, was at 104 days of age and for the genotype PM92007 silage was at 111 days of age.
\end{abstract}

Keywords: sunflower, age of harvest, cutivar, in vitro, dry matter, digestibility, $p H$

Recebido para publicação em 14 de setembro de 2004

Recebido para publicação, após modificações, em 5 de janeiro de 2005

*Autor para correspondência (corresponding author)

E-mail: sandra@vet.ufmg.br 


\section{INTRODUÇÃO}

No Brasil Central, a produção de forrageiras está condicionada a fatores climáticos. Durante o verão, ocorre aumento da disponibilidade de forragens atribuído aos fatores climáticos favoráveis. Na época seca do ano, há baixa disponibilidade de forragens para alimentação animal, face às baixas temperaturas e pluviosidades registradas nessa época. Uma das alternativas para minimizar os efeitos dessa sazonalidade na produção de forrageiras para alimentação animal é o uso de silagens.

O girassol é uma boa alternativa para áreas secas, em razão de sua tolerância à escassez de água, permitindo cultivo de baixo risco na safrinha e o uso de terras que ficariam ociosas após a colheita do milho ou do sorgo (Zago e Pozar, 1991).

O objetivo do presente experimento foi avaliar as características agronômicas e a composição bromatológica das silagens de quatro genótipos de girassol - M742, MG4, PM92007 e VDH483 - utilizados para produção de grãos, colhidos em cinco diferentes estádios de maturação - 90, 97, 104, 111 e 118 dias após o plantio.

\section{MATERIAL E MÉTODOS}

Foram plantados, colhidos e ensilados quatro genótipos de girassol (Helianthus annus L) nas dependências da EMBRAPA Milho e Sorgo, localizada no $\mathrm{km} 65$ da MG 424, no município de Sete Lagoas, Minas Gerais. O plantio foi realizado em 23/03/1999 e as colheitas, em 21/06, 28/06, 05/07, 12/07 e 19/07/1999.

O plantio foi feito em canteiros (ao acaso no campo), adotando-se cinco idades de corte e três repetições por genótipo. Os tratamentos foram realizados conforme esquema: T1- plantas colhidas após 90 dias após o plantio, T2- plantas colhidas após 97 dias após o plantio, T3- plantas colhidas após 104 dias após o plantio, T4plantas colhidas após 111 dias após o plantio, e T5- plantas colhidas após 118 dias após o plantio.

Após a amostragem da forragem fresca (três amostras de cada material) e imediatamente depois do corte, a forragem fresca foi picada em partículas de $2 \mathrm{~cm}$, utilizando-se uma picadeira estacionária Nogueira, modelo DPM-4, sendo o material homogeneizado para ensilagem. Foram utilizados 60 silos experimentais confeccionados em PVC, com $500 \mathrm{~mm}$ de comprimento e $100 \mathrm{~mm}$ de diâmetro, com peso vazio conhecido e capacidade para $2 \mathrm{~kg}$ de forragem fresca. A forragem foi compactada com pêndulo de madeira. Os silos, fechados com tampas de PVC dotadas de válvulas tipo "Bunsen" e lacrados com fita adesiva, foram pesados e abertos 72 dias após o fechamento.

O material amostrado no momento da ensilagem destinou-se a avaliações do material original, seguindo os procedimentos de rotina para experimentos dessa natureza.

Após 72 dias da ensilagem, os silos foram novamente pesados e abertos, sendo o seu conteúdo retirado e homogeneizado em balde plástico. Parte da silagem foi amostrada e congelada, outra parte foi seca em estufa de ventilação forçada e acondicionada da mesma forma descrita para o material original. $\mathrm{O}$ restante do material foi prensado em prensa hidráulica Carver, modelo C, para extração do suco da silagem. Parte desse material foi imediatamente utilizada para avaliação de $\mathrm{pH}$, utilizando-se um potenciômetro Beckman Expandomatic SS-2. A análise de nitrogênio amoniacal foi feita pelo método da destilação com óxido de magnésio e cloreto de cálcio (Official..., 1980). A outra parte do suco, 10ml, foi tratada com $2 \mathrm{ml}$ de ácido metafosfórico, congelada e posteriormente utilizada para dosagem de ácidos orgânicos da silagem. Posteriormente esse material foi descongelado, centrifugado e filtrado. A análise foi feita por cromatografia gasosa, em aparelho "Varian, modelo 2485", usando coluna de vidro de $2 \mathrm{~m}$ de comprimento e diâmetro de $1 / 8$ de polegadas com Cromosorb 101 (80-101 mesh).

Nas amostras pré-secas dos materiais e das silagens, determinaram-se os teores de matéria seca (MS), a $105^{\circ} \mathrm{C}$ (Official..., 1980), proteína bruta (PB) pelo método de Dumas (1830), citado por James (1995), extrato etéreo (EE) pelo processo Soxlet, segundo Official... (1995), carboidratos solúveis em álcool (Bailey, 1967), fibra em detergente neutro (FDN), fibra em detergente ácido (FDA), hemicelulose, celulose, lignina (Van Soest, 1994) e digestibilidade in 
vitro da matéria seca (DIVMS) (Tilley e Terry, 1963).

$\mathrm{O}$ experimento foi montado em um delineamento inteiramente ao acaso, em esquema fatorial $4 \times 5$ (4 genótipos, 5 idades) com três repetições. Utilizou-se o teste SNK para comparação entre as médias do desdobramento da interação genótipo versus idade $(\mathrm{P}<0,05)$. Para as análises, foi utilizado o pacote estatístico SAEG 8.0 (Sistema..., 1997).

\section{RESULTADOS E DISCUSSÃO}

Na Tab. 1 estão os resultados observados para os teores de MS, PB, EE, CHO e DIVMS no material original e nas silagens. A MS aumentou de $12,8 \%$ para $42,2 \%$ no material original e de $11,1 \%$ para $40,2 \%$ nas silagens. Tanto no material original quanto na silagem, os teores de MS aumentaram com o avanço do estádio de maturação da planta. Segundo Gonçalves e Tomich (1999), a confeç̧ão de silagens de girassol com baixo conteúdo de matéria seca, além de ser um fator limitante para a sua produção, é de difícil solução, pois o momento ideal para a ensilagem de girassol é na fase chamada "R9", ou seja, quando a planta atinge sua maturidade fisiológica. Mesmo nessa fase, com os aquênios apresentando $14 \%$ a $18 \%$ de MS, a parte vegetativa da planta ainda apresenta alta porcentagem de umidade (de $45 \%$ a $47 \%$ ). Tal fato explica a presença de teores de matéria seca abaixo daqueles preconizados por Paiva (1976) e McDonald et. al (1991), tornando-se evidente a necessidade de uma classificação específica para a silagem de girassol, para que essa possa expressar melhor a sua qualidade.

Os valores de $\mathrm{PB}$ encontrados variaram de $8,3 \%$ a $12,7 \%$ no material original e de $9,4 \%$ a $12,9 \%$ na silagem. Foram observadas diferenças $(\mathrm{P}<0,05)$ com o avanço do estádio de maturação da planta e entre os genótipos dentro da idade de corte, tanto para os materiais originais quanto para as silagens. Segundo Church (1988), a dieta ou o alimento dos ruminantes deve conter pelo menos 7\% de PB para fornecer nitrogênio suficiente para o desenvolvimento normal das bactérias ruminais, permitindo com isso uma fermentação eficiente. Esse valor foi alcançado em todos os genótipos, tanto para o material original quanto para a silagem.

Os teores de extrato etéreo do material original e das silagens variaram de $2,3 \%$ a $13,3 \%$ no material original e de $3,0 \%$ a $17,9 \%$ na silagem. Rodriguez et al. (1999) observaram que, à medida que aumentou a participação de capítulos na silagem de girassol, houve aumento $(\mathrm{P}<0,05)$ no teor de extrato etéreo.

Os teores de carboidratos solúveis, tanto para a silagem quanto para o material original, diminuíram $(\mathrm{P}<0,05)$ com o avanço do estádio de maturação da planta, sendo que os maiores valores foram obtidos na idade de corte de 97 dias. Entre o material original e a silagem, observou-se redução $(\mathrm{P}<0,05)$ nos teores de carboidratos solúveis, o que demonstra extensa fermentação dessa fração ao longo do processo fermentativo para todas as idades de corte em todos os genótipos avaliados.

Com o avanço do estádio de maturação da planta, houve diminuição $(\mathrm{P}<0,05)$ dos valores de digestibilidade in vitro para o material original e para as silagens. De acordo com McDonald et al. (1991), o declínio na digestibilidade com o avanço da maturidade da planta é resultado principalmente do aumento do conteúdo de carboidratos estruturais, menos digestíveis que os componentes solúveis da planta. A variação encontrada para DIVMS foi de $46,3 \%$ a $64,0 \%$ para o material original e $44,0 \%$ a $59,4 \%$ para a silagem.

Na Tab. 2 são mostrados os resultados dos teores de FDN, FDA, hemicelulose, celulose e lignina (\% MS) no material original e nas silagens. No primeiro, os valores de FDN variaram de $47,7 \%$ para o cultivar M742, na segunda idade de corte, a $58,4 \%$ para o cultivar MG4, na idade de corte de 111 dias. Na silagem, a variação foi de $43,1 \%$ para o cultivar VDH483, na segunda idade de corte, e $52,9 \%$ para o cultivar PM92007, na idade de corte de 118 dias. Verificou-se redução $(\mathrm{P}<0,05)$ nos valores de FDN com a ensilagem, sugerindo que houve hidrólise dos componentes estruturais durante a fermentação. Essa redução não foi observada por Tomich (1999), enquanto Stehling (2001) observou redução em um cultivar. 
Tabela 1. Matéria seca (MS), proteína bruta (PB), extrato etéreo (EE), carboidratos solúveis (CHO) e digestibilidade in vitro da matéria seca em porcentagem do material original (MO) e das silagens (SIL) de quatro genótipos de girassol, ensilados em diferentes idades de corte

\begin{tabular}{|c|c|c|c|c|c|}
\hline \multicolumn{6}{|c|}{ Idade de corte (dias) } \\
\hline Cultivar & 90 & 97 & 104 & 111 & 118 \\
\hline & \multicolumn{5}{|c|}{ MS } \\
\hline M742 (MO) & $14,6 \mathrm{Da} \alpha$ & $20,3 \mathrm{Ca} \alpha$ & $20,7 \mathrm{Ca} \alpha$ & $27,8 \mathrm{Ba} \alpha$ & $42,2 \mathrm{Aa} \alpha$ \\
\hline MG4 (MO) & $14,5 \mathrm{Da} \alpha$ & $18,1 \mathrm{Ca} \alpha$ & $20,0 \mathrm{Ca} \alpha$ & $25,0 \mathrm{Ba} \alpha$ & $37,1 \mathrm{Ab} \alpha$ \\
\hline PM92007 (MO) & $12,7 \mathrm{Da} \alpha$ & $16,5 \mathrm{Ca} \alpha$ & $17,0 \mathrm{Ca} \alpha$ & $20,5 \mathrm{Ba} \alpha$ & $29,7 \mathrm{Ad} \alpha$ \\
\hline VDH483 (MO) & $13,0 \mathrm{Da} \alpha$ & $18,5 \mathrm{Ca} \alpha$ & $19,7 \mathrm{Ca} \alpha$ & $25,1 \mathrm{Ba} \alpha$ & 33,3 Ac $\alpha$ \\
\hline M742 (SIL) & $14,7 \mathrm{Da} \alpha$ & $17,6 \mathrm{Cda} \alpha$ & $20,4 \mathrm{Ca} \alpha$ & $25,9 \mathrm{Ba} \alpha$ & $40,2 \mathrm{Aa} \alpha$ \\
\hline MG4 (SIL) & $13,4 \mathrm{Da} \alpha$ & $15,8 \mathrm{Da} \alpha$ & $19,1 \mathrm{Cab} \alpha$ & $24,8 \mathrm{Ba} \alpha$ & $34,6 \mathrm{Ab} \alpha$ \\
\hline PM92007 (SIL) & $11,1 \mathrm{da} \alpha$ & $14,3 \mathrm{Ca} \alpha$ & $16,3 \mathrm{BCb} \alpha$ & $19,1 \mathrm{Bb} \alpha$ & $27,5 \mathrm{Ad} \alpha$ \\
\hline \multirow[t]{2}{*}{ VDH483 (SIL) } & $13,9 \mathrm{Da} \alpha$ & $15,4 \mathrm{Cda} \beta$ & $18,3 \mathrm{Cab} \alpha$ & $26,1 \mathrm{Ba} \alpha$ & 31,6 Ac $\alpha$ \\
\hline & \multicolumn{5}{|c|}{ PB } \\
\hline M742 (MO) & $11,3 \mathrm{Aa} \beta$ & $11,6 \mathrm{Aa} \alpha$ & $10,3 \mathrm{Aa} \beta$ & $10,1 \mathrm{Aa} \alpha$ & $11,0 \mathrm{Aab} \alpha$ \\
\hline MG4 (MO) & $12,3 \mathrm{Aa} \alpha$ & $10,7 \mathrm{Bab} \alpha$ & $10,2 \mathrm{Ba} \alpha$ & $9,4 \mathrm{Ba} \alpha$ & $9,7 \mathrm{Bb} \alpha$ \\
\hline PM92007 (MO) & $12,7 \mathrm{Aa} \alpha$ & $9,7 \mathrm{Bb} \alpha$ & $8,3 \mathrm{Cb} \beta$ & $10,1 \mathrm{Bab} \alpha$ & $10,6 \mathrm{Bab} \alpha$ \\
\hline VDH483 (MO) & $12,0 \mathrm{Aa} \alpha$ & $9,8 \mathrm{Bb} \alpha$ & $10,0 \mathrm{Ba} \alpha$ & $10,3 \mathrm{Ba} \alpha$ & $12,0 \mathrm{Aa} \alpha$ \\
\hline M742 (SIL) & $12,9 \mathrm{Aa} \alpha$ & $11,0 \mathrm{Ba} \alpha$ & $11,7 \mathrm{Ba} \alpha$ & $11,1 \mathrm{Ba} \alpha$ & $11,0 \mathrm{Ba} \alpha$ \\
\hline MG4 (SIL) & $11,2 \mathrm{Ab} \alpha$ & $10,2 \mathrm{Aa} \alpha$ & $9,7 \mathrm{Bc} \alpha$ & $9,5 \mathrm{Bb} \alpha$ & $9,4 \mathrm{Bb} \alpha$ \\
\hline PM92007 (SIL) & $12,3 \mathrm{Aab} \alpha$ & $10,2 \mathrm{Ba} \alpha$ & $10,2 \mathrm{Bbc} \alpha$ & $10,5 \mathrm{Bab} \alpha$ & $10,6 \mathrm{Ba} \alpha$ \\
\hline \multirow[t]{2}{*}{ VDH483 (SIL) } & $10,9 \mathrm{Ab} \alpha$ & $10,8 \mathrm{Aa} \alpha$ & $11,2 \mathrm{Aab} \alpha$ & $10,3 \mathrm{Aab} \alpha$ & $11,1 \mathrm{Aa} \alpha$ \\
\hline & \multicolumn{5}{|c|}{ EE } \\
\hline M742 (MO) & $7,2 \mathrm{Ca} \alpha$ & $11,4 \mathrm{Aba} \alpha$ & $12,1 \mathrm{Aa} \alpha$ & $9,4 \mathrm{BCb} \beta$ & $9,6 \mathrm{BCb} \beta$ \\
\hline MG4 (MO) & $3,1 \mathrm{Cbc} \alpha$ & $4,6 \mathrm{Cc} \alpha$ & $8,4 \mathrm{Abb} \beta$ & $10,3 \mathrm{Ab} \alpha$ & $7,6 \mathrm{Bb} \beta$ \\
\hline PM92007 (MO) & $2,3 \mathrm{Cc} \beta$ & $3,1 \mathrm{Cc} \beta$ & $6,1 \mathrm{Bc} \beta$ & $9,6 \mathrm{Ab} \beta$ & $8,4 \mathrm{Ab} \alpha$ \\
\hline VDH483 (MO) & $5,0 \mathrm{Cb} \beta$ & $7,5 \mathrm{Bb} \beta$ & $12,4 \mathrm{Aa} \beta$ & $13,3 \mathrm{Aa} \beta$ & $11,8 \mathrm{Aa} \beta$ \\
\hline M742 (SIL) & $8,1 \mathrm{Ca} \alpha$ & $12,0 \mathrm{Ba} \alpha$ & $12,6 \mathrm{Abb} \alpha$ & $14,9 \mathrm{Aa} \alpha$ & $13,1 \mathrm{Abb} \alpha$ \\
\hline MG4 (SIL) & $3,0 \mathrm{Bb} \alpha$ & $4,5 \mathrm{Bb} \alpha$ & $10,5 \mathrm{Ab} \alpha$ & $11,7 \mathrm{Ab} \alpha$ & $11,8 \mathrm{Ab} \alpha$ \\
\hline PM92007 (SIL) & $7,7 \mathrm{Ca} \alpha$ & $6,13 \mathrm{Cb} \alpha$ & $10,8 \mathrm{Bb} \alpha$ & $14,8 \mathrm{Aa} \alpha$ & $9,7 \mathrm{Bc} \alpha$ \\
\hline \multirow[t]{2}{*}{ VDH483 (SIL) } & $9,8 \mathrm{Ca} \alpha$ & $12,9 \mathrm{Ba} \alpha$ & $18,0 \mathrm{Aa} \alpha$ & $15,8 \mathrm{Aa} \alpha$ & $17,6 \mathrm{Aa} \alpha$ \\
\hline & \multicolumn{5}{|c|}{$\mathrm{CHO}$} \\
\hline M742 (MO) & 7,2Aba & $8,5 \mathrm{Bc} \alpha$ & $5,5 \mathrm{Cc} \alpha$ & $2,8 \mathrm{Dc} \alpha$ & $1,2 \mathrm{Ec} \alpha$ \\
\hline MG4 (MO) & $9,5 \mathrm{Ba} \alpha$ & $13,0 \mathrm{Aa} \alpha$ & $9,2 \mathrm{Ca} \alpha$ & $5,0 \mathrm{Db} \alpha$ & $1,7 \mathrm{~Eb} \alpha$ \\
\hline PM92007 (MO) & $6,9 \mathrm{Dc} \alpha$ & $14,0 \mathrm{Aa} \alpha$ & $8,6 \operatorname{cb} \alpha$ & $9,3 \mathrm{Ba \alpha}$ & $2,1 \mathrm{Ea} \alpha$ \\
\hline VDH483 (MO) & $5,4 \mathrm{Ba} \alpha$ & $11,5 \mathrm{Ab} \alpha$ & $4,6 \mathrm{Cd} \alpha$ & $2,9 \mathrm{Dc} \alpha$ & $2,1 \mathrm{Ea} \alpha$ \\
\hline M742 (SIL) & $0,3 \mathrm{Aa} \beta$ & $0,3 \mathrm{Ab} \beta$ & $0,1 \mathrm{Aa} \beta$ & $0,1 \mathrm{Ab} \beta$ & $0,2 \mathrm{Aa} \beta$ \\
\hline MG4 (SIL) & $0,4 \mathrm{Aa} \beta$ & $0,4 \mathrm{Aab} \beta$ & $0,2 \mathrm{ABa} \beta$ & $0,1 \mathrm{Bb} \beta$ & $0,1 \mathrm{Ba} \beta$ \\
\hline PM92007 (SIL) & $0,5 \mathrm{Aba} \beta$ & $0,6 \mathrm{Aa} \beta$ & $0,3 \mathrm{BCa} \beta$ & $0,4 \mathrm{ABCa} \beta$ & $0,2 \mathrm{Ca} \beta$ \\
\hline \multirow[t]{2}{*}{ VDH483 (SIL) } & $0,4 \mathrm{Aa} \beta$ & $0,5 \mathrm{Aab} \beta$ & $0,2 \mathrm{Ba} \beta$ & $0,1 \mathrm{Bb} \beta$ & $0,1 \mathrm{Ba} \beta$ \\
\hline & \multicolumn{5}{|c|}{ DIVMS } \\
\hline M742 (MO) & $57,8 \mathrm{Aa} \alpha$ & 55,8 Ac $\alpha$ & $55,0 \mathrm{Aa} \alpha$ & $54,0 \mathrm{Aa} \alpha$ & $48,5 \mathrm{Ba} \alpha$ \\
\hline MG4 (MO) & $58,5 \mathrm{Ba} \alpha$ & $63,9 \mathrm{Aa} \alpha$ & $53,8 \mathrm{Ca} \alpha$ & $46,9 \mathrm{Db} \alpha$ & $46,3 \mathrm{Da} \alpha$ \\
\hline PM92007 (MO) & $58,7 \mathrm{Aa} \alpha$ & $57,4 \mathrm{Abc} \alpha$ & $50,5 \mathrm{Bca} \beta$ & $52,1 \mathrm{Ba} \alpha$ & $46,9 \mathrm{Ca} \alpha$ \\
\hline VDH483 (MO) & $57,6 \mathrm{Aa} \alpha$ & $61,0 \mathrm{Aab} \alpha$ & $53,5 \mathrm{Ba}$ & $50,8 \mathrm{Ba} \alpha$ & $49,6 \mathrm{Ba} \alpha$ \\
\hline M742 (SIL) & $58,1 \mathrm{Aa} \alpha$ & $57,6 \mathrm{Aab} \alpha$ & $56,5 \mathrm{Aa} \alpha$ & $50,4 \mathrm{Ba} \alpha$ & $49,6 \mathrm{Ba \alpha}$ \\
\hline MG4 (SIL) & $59,3 \mathrm{Aa} \alpha$ & $59,4 \mathrm{Aa} \beta$ & $56,0 \mathrm{Aa} \alpha$ & $49,1 \mathrm{Ba} \alpha$ & $43,9 \mathrm{Cb} \alpha$ \\
\hline PM92007 (SIL) & $57,8 \mathrm{Aa} \alpha$ & $54,8 \mathrm{ABab} \alpha$ & $55,8 \mathrm{ABa} \alpha$ & $51,1 \mathrm{BCa} \alpha$ & $48,5 \mathrm{Cab} \alpha$ \\
\hline VDH483 (SIL) & $59,3 \mathrm{Aa} \alpha$ & $54,2 \mathrm{Bb} \beta$ & $55,8 \mathrm{ABa} \alpha$ & $47,2 \mathrm{Ca} \alpha$ & $46,7 \mathrm{Cab} \alpha$ \\
\hline
\end{tabular}

Letras maiúsculas distintas na mesma linha indicam diferenças $(\mathrm{P}<0,05)$ para cada híbrido nas diferentes idades de corte.

Letras minúsculas distintas na mesma coluna indicam diferenças $(\mathrm{P}<0,05)$ entre os híbridos (material original ou silagem) na mesma idade de corte.

Letras gregas distintas na mesma coluna indicam diferenças $(\mathrm{P}<0,05)$ entre a silagem e o material original para cada uma das frações fibrosas MS, CV: 8,5\%; PB, CV: 6,6\%; EE, CV: 10,7\%; CHO, CV:35,3\%; DIVMS, CV:4,3\%. Teste SNK (P<0,05). 
Tabela 2. Fibra em detergente neutro (FDA), fibra em detergente ácido (FDA), hemicelulose, celulose, lignina em porcentagem da matéria seca do material original (MO) e das silagens (SIL) de quatro genótipos de girassol, ensilados em diferentes idades de corte

\begin{tabular}{|c|c|c|c|c|c|}
\hline \multicolumn{6}{|c|}{ Idade de corte (dias) } \\
\hline Cultivar & 90 & 97 & 104 & 111 & 118 \\
\hline & \multicolumn{5}{|c|}{ FDN } \\
\hline M742 (MO) & $53,5 \mathrm{Aa} \alpha$ & $47,7 \mathrm{Ba} \alpha$ & $48,0 \mathrm{Bb} \alpha$ & $53,1 \mathrm{ABca}$ & $52,2 \mathrm{Abca}$ \\
\hline MG4 (MO) & $53,8 \mathrm{Ba} \alpha$ & $48,7 \mathrm{Ca} \alpha$ & $54,9 \mathrm{Aba} \alpha$ & $58,5 \mathrm{Aa} \alpha$ & 58,2 Aa $\alpha$ \\
\hline PM92007 (MO) & $53,0 \mathrm{Aba} \alpha$ & $50,9 \mathrm{Aba} \alpha$ & $52,3 \mathrm{Aba}$ & $50,4 \mathrm{Bc} \alpha$ & $55,2 \mathrm{Aab} \alpha$ \\
\hline VDH483 (MO) & $54,1 \mathrm{Aba} \alpha$ & $47,7 \mathrm{Ca} \alpha$ & 51,3 Bca $\alpha$ & $56,3 \mathrm{Aab} \alpha$ & $50,4 \mathrm{BCc} \alpha$ \\
\hline M742 (SIL) & $47,4 \mathrm{Aab} \beta$ & $45,1 \mathrm{Aab} \alpha$ & 45,9Aa $\alpha$ & $46,1 \mathrm{Ab} \beta$ & $48,5 \mathrm{Ab} \beta$ \\
\hline MG4 (SIL) & $48,3 \mathrm{Bab} \beta$ & $45,3 \mathrm{Bb} \alpha$ & $46,5 \mathrm{Ba} \beta$ & $51,6 \mathrm{Aa} \beta$ & $52,5 \mathrm{Aa} \beta$ \\
\hline PM92007 (SIL) & $50,5 \mathrm{Aba} \alpha$ & $48,9 \mathrm{Ba} \alpha$ & $48,4 \mathrm{Ba} \beta$ & $48,1 \mathrm{Bb} \alpha$ & $52,9 \mathrm{Aa} \alpha$ \\
\hline \multirow[t]{2}{*}{ VDH483 (SIL) } & $45,2 \mathrm{Ab} \beta$ & $43,1 \mathrm{Ab} \beta$ & $44,8 \mathrm{Aa} \beta$ & $45,8 \mathrm{Ab} \beta$ & $43,8 \mathrm{Ac} \beta$ \\
\hline & \multicolumn{5}{|c|}{ FDA } \\
\hline M742 (MO) & $36,5 \mathrm{Abb} \alpha$ & $34,8 \mathrm{Bb} \alpha$ & $35,9 \mathrm{Abb} \alpha$ & $36,8 \mathrm{Abb} \alpha$ & $39,1 \mathrm{Aab} \alpha$ \\
\hline MG4 (MO) & $37,3 \mathrm{Bb} \alpha$ & $34,3 \mathrm{Bb} \alpha$ & $37,1 \mathrm{Bab} \alpha$ & 41,3Aa $\alpha$ & $40,6 \mathrm{Aa} \alpha$ \\
\hline PM92007 (MO) & $41,4 \mathrm{Aa} \alpha$ & $39,2 \mathrm{ABa} \alpha$ & $39,8 \mathrm{Bb} \alpha$ & $37,3 \mathrm{Bb} \alpha$ & $41,2 \mathrm{Aa} \alpha$ \\
\hline VDH483 (MO) & $37,3 \mathrm{Ab} \alpha$ & $34,7 \mathrm{Ab} \alpha$ & $34,4 \mathrm{Ab} \alpha$ & $36,9 \mathrm{Ab} \alpha$ & $36,6 \mathrm{Ab} \alpha$ \\
\hline M742 (SIL) & $36,8 \mathrm{Ab} \alpha$ & $34,8 \mathrm{Ab} \alpha$ & $35,1 \mathrm{Aab} \alpha$ & $36,1 \mathrm{Aa} \alpha$ & $38,6 \mathrm{Aa} \alpha$ \\
\hline MG4 (SIL) & $37,6 \mathrm{Ab} \alpha$ & $34,5 b \alpha$ & $38,2 \mathrm{Aa} \beta$ & $38,2 \mathrm{Aa} \beta$ & $39,9 \mathrm{Aa} \alpha$ \\
\hline PM92007 (SIL) & $40,5 \mathrm{Aa} \alpha$ & $38,7 \mathrm{Aba} \alpha$ & $37,4 \mathrm{Aba} \alpha$ & $35,7 \mathrm{Ba \alpha}$ & $40,4 \mathrm{Aa} \alpha$ \\
\hline \multirow[t]{2}{*}{ VDH483 (SIL) } & $35,1 \mathrm{Aab} \alpha$ & $33,5 \mathrm{Ab \alpha}$ & $33,3 \mathrm{Ab} \alpha$ & $36,1 \mathrm{Aa} \alpha$ & $34,1 \mathrm{Ab} \alpha$ \\
\hline & \multicolumn{5}{|c|}{ Hemicelulose } \\
\hline M742 (MO) & $17,1 \mathrm{Aa} \alpha$ & $12,9 \mathrm{Bab} \alpha$ & $12,1 \mathrm{Bb} \alpha$ & $16,3 \mathrm{Ab} \alpha$ & $13,2 \mathrm{Bb} \alpha$ \\
\hline MG4 (MO) & $16,4 \mathrm{Aa} \alpha$ & $14,3 \mathrm{Ba} \alpha$ & $17,8 \mathrm{Aa} \alpha$ & $17,2 \mathrm{Ab} \alpha$ & $17,6 \mathrm{Aa} \alpha$ \\
\hline PM92007 (MO) & $11,6 \mathrm{Bb} \alpha$ & $11,7 \mathrm{Bb} \alpha$ & $12,4 \mathrm{ABb} \alpha$ & $13,2 \mathrm{Abc} \alpha$ & $14,1 \mathrm{Ab} \alpha$ \\
\hline VDH483 (MO) & $16,6 \mathrm{Ba} \alpha$ & $13,0 \mathrm{Cab} \alpha$ & $16,9 \mathrm{Ba} \alpha$ & 19,1Аa $\alpha$ & $13,8 \mathrm{Cb} \alpha$ \\
\hline M742 (SIL) & $10,6 \mathrm{Aa} \beta$ & $10,4 \mathrm{Aa} \beta$ & $10,8 \mathrm{Aa} \alpha$ & $10,1 \mathrm{Ab} \beta$ & $9,9 \mathrm{Ab} \beta$ \\
\hline MG4 (SIL) & $10,7 \mathrm{Ba} \beta$ & $10,8 \mathrm{Ba} \beta$ & $11,8 \mathrm{Aba} \beta$ & $13,4 \mathrm{Aa} \beta$ & $12,5 \mathrm{Aba} \beta$ \\
\hline PM92007 (SIL) & $1,0 \mathrm{Ba} \beta$ & $10,2 \mathrm{Ba} \beta$ & $11,1 \mathrm{Aba} \alpha$ & $12,4 \mathrm{Aa} \alpha$ & $12,5 \mathrm{Aa} \beta$ \\
\hline \multirow[t]{2}{*}{ VDH483 (SIL) } & $10,1 \mathrm{Aa} \beta$ & $9,5 \mathrm{Aa} \beta$ & $11,4 \mathrm{Aa} \beta$ & $9,7 \mathrm{Ab} \beta$ & $9,7 \mathrm{Ab} \beta$ \\
\hline & \multicolumn{5}{|c|}{ Celulose } \\
\hline M742 (MO) & $26,1 \mathrm{Aa} \alpha$ & $26,2 \mathrm{Aa} \alpha$ & $27,1 \mathrm{Aa} \alpha$ & $26,9 \mathrm{Aa} \alpha$ & $29,7 \mathrm{Aa} \alpha$ \\
\hline MG4 (MO) & $27,3 \mathrm{Aa} \alpha$ & $24,2 \mathrm{Aa} \alpha$ & $26,2 \mathrm{Aa} \alpha$ & $28,7 \mathrm{Aa} \alpha$ & $30,9 \mathrm{Aa} \alpha$ \\
\hline PM92007 (MO) & $29,4 \mathrm{Aa} \alpha$ & $29,7 \mathrm{Aa} \alpha$ & $30,4 \mathrm{Aa} \alpha$ & $27,4 \mathrm{Aa} \alpha$ & $31,4 \mathrm{Aa} \alpha$ \\
\hline VDH483 (MO) & $26,3 \mathrm{Aa} \alpha$ & $25,0 \mathrm{Aa} \alpha$ & $25,4 \mathrm{Aa} \alpha$ & $25,8 \mathrm{Aa} \alpha$ & $26,0 \mathrm{Aa} \alpha$ \\
\hline M742 (SIL) & $28,3 \mathrm{Aa} \alpha$ & $27,3 \mathrm{Aa} \alpha$ & $27,9 \mathrm{Aa} \alpha$ & $28,7 \mathrm{Aa} \alpha$ & $30,6 \mathrm{Aa} \alpha$ \\
\hline MG4 (SIL) & 29,6 Aa $\alpha$ & $27,5 \mathrm{Aa} \alpha$ & $27,8 \mathrm{Aa} \alpha$ & 28,1 Aa $\alpha$ & $31,5 \mathrm{Aa} \alpha$ \\
\hline PM92007 (SIL) & $31,8 \mathrm{Aa} \alpha$ & $21,4 \mathrm{Ba} \beta$ & $29,8 \mathrm{Aa} \alpha$ & $28,6 \mathrm{Aa} \alpha$ & $33,1 \mathrm{Aa} \alpha$ \\
\hline \multirow[t]{2}{*}{ VDH483 (SIL) } & $26,4 \mathrm{Aa} \alpha$ & $25,3 \mathrm{Aa} \alpha$ & $25,7 \mathrm{Aa} \alpha$ & $28,0 \mathrm{Aa} \alpha$ & $26,4 \mathrm{Aa} \alpha$ \\
\hline & \multicolumn{5}{|c|}{ Lignina } \\
\hline M742 (MO) & $10,1 \mathrm{Ab} \alpha$ & $8,6 \mathrm{Aa} \alpha$ & $9,0 \mathrm{Aa} \alpha$ & $10,1 \mathrm{Ab} \alpha$ & $9,4 \mathrm{Aa} \alpha$ \\
\hline MG4 (MO) & $9,3 \mathrm{Bb} \alpha$ & $9,6 \mathrm{Ba} \alpha$ & $10,4 \mathrm{Ba} \alpha$ & $12,0 \mathrm{Aa} \alpha$ & $9,4 \mathrm{Ba} \alpha$ \\
\hline PM92007 (MO) & $11,6 \mathrm{Aa} \alpha$ & $9,6 \mathrm{Ba} \alpha$ & $9,6 \mathrm{Ba} \alpha$ & $10,2 b b \alpha$ & $9,7 \mathrm{Ba} \alpha$ \\
\hline VDH483 (MO) & $11,4 \mathrm{Aa} \alpha$ & $9,9 \mathrm{Aba} \alpha$ & $9,0 \mathrm{Ba} \alpha$ & $10,6 \mathrm{ABb} \alpha$ & $9,7 \mathrm{Ba} \alpha$ \\
\hline M742 (SIL) & $8,3 \mathrm{Aa} \beta$ & $7,3 \mathrm{Aa} \beta$ & $7,0 \mathrm{Aa} \beta$ & $7,3 \mathrm{Aa} \beta$ & $8,0 \mathrm{Aa} \beta$ \\
\hline MG4 (SIL) & $8,0 \mathrm{Aba} \beta$ & $7,2 \mathrm{ABa} \beta$ & $6,8 \mathrm{Ba} \beta$ & $8,0 \mathrm{Aba} \beta$ & 8,6 Aa $\alpha$ \\
\hline PM92007 (SIL) & $8,7 \mathrm{Aa} \beta$ & $8,5 \mathrm{Aa} \alpha$ & $7,6 \mathrm{Aa} \beta$ & $7,4 \mathrm{Aa} \beta$ & $8,0 \mathrm{Aa} \beta$ \\
\hline VDH483 (SIL) & $8,6 \mathrm{Aa} \beta$ & $8,0 \mathrm{Aa} \beta$ & $7,5 \mathrm{Aa} \beta$ & $8,2 \mathrm{Aa} \beta$ & $7,4 \mathrm{Aa} \beta$ \\
\hline
\end{tabular}

Letras maiúsculas distintas na mesma linha indicam diferenças $(\mathrm{P}<0,05)$ para cada híbrido nas diferentes idades de corte.

Letras minúsculas distintas na mesma coluna indicam diferenças $(\mathrm{P}<0,05)$ entre os híbridos (material original ou silagem) na mesma idade de corte.

Letras gregas distintas na mesma coluna indicam diferenças $(\mathrm{P}>0,05)$ entre a silagem e o material original para cada uma das frações fibrosas FDN, CV:4,2; hemicelulose, CV: 7,9; celulose, CV: 14,5; lignina, CV:9,3. Teste $\mathrm{SNK}(\mathrm{P}<0,05)$. 
Quanto aos valores de FDA, no material original, houve variação de $34,3 \%$ a $41,4 \%$ e, nas silagens, de $33,3 \%$ a $40,6 \%$. Com o avançar do estádio de maturação da planta, houve aumento $(\mathrm{P}<0,05)$ nos teores de FDA, sendo os maiores valores observados na idade de 118 dias, tanto para o material original quanto para a silagem. Quanto ao FDA, não houve diferença $(\mathrm{P}>0,05)$ entre o material original e a silagem.

Com relação à hemicelulose, não houve variação $(\mathrm{P}>0,05)$ dos valores com o avanço da idade de corte para o material original e para as silagens. No material original, houve aumento $(\mathrm{P}<0,05)$ de $11,6 \%$ a $19,1 \%$, enquanto nas silagens os teores aumentaram $(\mathrm{P}<0,05)$ de $9,5 \%$ a $13,4 \%$. Houve diminuição $(\mathrm{P}<0,05)$ nos teores de hemicelulose com a ensilagem. Essa diminuição foi observada para todos os genótipos, exceto para os cultivares M742, na idade de 104 dias e o cultivar PM92007, na idade de 111 dias.

Os teores de celulose, no material original, aumentaram de $24,2 \%$ para $31,4 \%$ para os genótipos MG4, na segunda idade de corte aos 97 dias, e PM92007, na idade de corte de 118 dias. Na silagem, o aumento $(\mathrm{P}<0,05)$ foi de $21,4 \%$ a $33,1 \%$ para o genótipo PM92007, na segunda e na quinta idade de corte, respectivamente. Não houve diferença $(\mathrm{P}>0,05)$ nos valores de celulose com o avanço do estádio de maturação da planta, bem como entre os genótipos na mesma idade de corte para o material original e para as silagens.

Não houve diferença $(\mathrm{P}>0,05)$ nos valores de hemicelulose com a ensilagem, exceto para o genótipo PM92007, na segunda idade de corte. Segundo Van Soest (1994), a celulose é um carboidrato estável dentro do silo, e as reduções dessa fração podem ocorrer normalmente em silos onde houver fermentação do tipo aeróbica, com formação de mofo.

Com relação à lignina, no material original, os teores aumentaram de $8,6 \%$ para $11,9 \%$ e, na silagem, a variação foi de $6,8 \%$ a $8,7 \%$. Para o material original, não houve variação nos valores de lignina para o genótipo M742 com o avanço do estádio de maturação, o mesmo não ocorrendo para os demais genótipos. Os menores valores foram observados para a quinta idade de corte. Nas silagens, não houve variação $(\mathrm{P}>0,05)$ nos teores de lignina com o avanço do estádio de maturação. Houve redução nos valores de lignina em todos os híbridos, exceto para os genótipos PM92007, na segunda idade de corte, e MG4 na quinta idade de corte, cujas reduções não foram significativas. Essas reduções podem estar ligadas ao método de determinação da lignina.

$\mathrm{Na}$ Tab. 3 são mostrados os teores de ácidos acétido, propiônico, butírico, lático, o pH e os teores de $\mathrm{NH}_{3} / \mathrm{NT}$ das silagens. Com relação ao ácido acético, não foram observadas diferenças $(\mathrm{P}>0,05)$ entre as idades de colheita e entre os genótipos, com exceção do genótipo PM92007, na idade de corte de 118 dias, que foi de $6,4 \%$.

Os valores de ácido propiônico aumentaram gradativamente com o estádio de maturação da planta em todos os genótipos. A variação ocorrida foi de $0,1 \%$ para o material PM92007, na idade de corte de 118 dias até $0,5 \%$ para o cultivar VDH483, na idade de corte de 118 dias. Os valores observados para ácido acético e propiônico indicam que as silagens de todos os genótipos apresentaram qualidade muito boa sob o aspecto de fermentação.

Os teores de ácido butírico variaram de 0,0\% para todos os cultivares, na quinta idade de corte, até 0,3 para o genótipo M742, na idade de corte de 90 dias. Não foi observada $(\mathrm{P}>0,05)$ diferença com o avanço do estádio de maturação tampouco entre os genótipos dentro da mesma idade de corte, exceto para o genótipo M742, na idade de corte de 90 dias. Verificou-se diminuição $(\mathrm{P}<0,05)$ nos valores de ácido butírico com o avanço do estádio de maturação da planta. $\mathrm{Na}$ idade de corte de 118 dias, não se observou a presença desse ácido em todos os genótipos. Segundo a classificação proposta por Paiva (1976) para o ácido butírico, as silagens de todos os genótipos, a partir da idade de corte de 97 dias, podem ser classificadas como sendo de muito boa qualidade sob o aspecto de fermentação. 
Tabela 3. Ácidos acético, propiônico, butírico e lático, nitrogênio amoniacal em porcentagem do nitrogênio total $\left(\mathrm{NH}_{3} / \mathrm{NT}\right)$ e $\mathrm{pH}$ das silagens de quatro genótipos de girassol ensilados em diferentes idades de corte

\begin{tabular}{|c|c|c|c|c|c|}
\hline \multicolumn{6}{|c|}{ Idade de corte (dias) } \\
\hline Cultivar & 90 & 97 & 104 & 111 & 118 \\
\hline & \multicolumn{5}{|c|}{ Ac. acético } \\
\hline M742 & $2,2 \mathrm{Aa}$ & $2,5 \mathrm{Aa}$ & $2,5 \mathrm{Aa}$ & 2,7Aa & $2,4 \mathrm{Ab}$ \\
\hline MG4 & $2,1 \mathrm{Ba}$ & $3,3 \mathrm{Aa}$ & $2,6 \mathrm{Aba}$ & $2,5 \mathrm{Aba}$ & $3,2 \mathrm{Ab}$ \\
\hline PM92007 & $3,0 \mathrm{Ba}$ & $2,6 \mathrm{Ba}$ & $2,3 \mathrm{Ba}$ & $2,6 \mathrm{Ba}$ & $6,4 \mathrm{Aa}$ \\
\hline \multirow[t]{2}{*}{ VDH483 } & $2,6 \mathrm{Aa}$ & $3,2 \mathrm{Aa}$ & $2,3 \mathrm{Aa}$ & $2,4 \mathrm{Aa}$ & $3,1 \mathrm{Ab}$ \\
\hline & \multicolumn{5}{|c|}{ Ac. propiônico } \\
\hline M742 & $0,1 \mathrm{Ba}$ & $0,2 \mathrm{Ba}$ & $0,2 \mathrm{Ba}$ & $0,3 \mathrm{Aa}$ & $0,4 \mathrm{Aab}$ \\
\hline MG4 & $0,1 \mathrm{Ba}$ & $0,2 \mathrm{Ba}$ & $0,1 \mathrm{Ba}$ & $0,3 \mathrm{Aa}$ & $0,4 \mathrm{Aab}$ \\
\hline PM92007 & $0,1 \mathrm{Ba}$ & $0,1 \mathrm{Ba}$ & $0,1 \mathrm{Ba}$ & $0,1 \mathrm{Bb}$ & $0,3 \mathrm{Ab}$ \\
\hline \multirow{2}{*}{ VDH483 } & $0,1 \mathrm{Ca}$ & $0,2 \mathrm{Ca}$ & $0,1 \mathrm{Ca}$ & $0,3 \mathrm{Ba}$ & $0,5 \mathrm{Aa}$ \\
\hline & \multicolumn{5}{|c|}{ Ac. butírico } \\
\hline M742 & $0,3 \mathrm{Aa}$ & $0,0 \mathrm{Ba}$ & $0,1 \mathrm{Ba}$ & $0,0 \mathrm{Ba}$ & $0,0 \mathrm{Ba}$ \\
\hline MG4 & $0,1 \mathrm{Ab}$ & $0,1 \mathrm{Aa}$ & $0,1 \mathrm{Aa}$ & $0,0 \mathrm{Aa}$ & $0,0 \mathrm{Aa}$ \\
\hline PM92007 & $0,1 \mathrm{Ab}$ & $0,1 \mathrm{Aa}$ & $0,0 \mathrm{Aa}$ & $0,0 \mathrm{Aa}$ & $0,0 \mathrm{Aa}$ \\
\hline \multirow[t]{2}{*}{ VDH483 } & $0,1 \mathrm{Ab}$ & $0,1 \mathrm{Aa}$ & $0,0 \mathrm{Aa}$ & $0,0 \mathrm{Aa}$ & $0,0 \mathrm{Aa}$ \\
\hline & \multicolumn{5}{|c|}{ Ac. lático } \\
\hline M742 & $11,8 \mathrm{Ab}$ & $13,6 \mathrm{Aa}$ & $10,9 \mathrm{Aa}$ & $6,2 \mathrm{C}$ & $8,2 \mathrm{Cb}$ \\
\hline MG4 & $15,7 \mathrm{Aa}$ & $10,6 \mathrm{Bbc}$ & $10,7 \mathrm{Cb}$ & $7,4 \mathrm{Cb}$ & $8,1 \mathrm{Cb}$ \\
\hline PM92007 & $14,6 \mathrm{Aa}$ & $12,6 \mathrm{Abab}$ & $10,9 \mathrm{ba}$ & $12,6 \mathrm{Aba}$ & $6,1 \mathrm{Cb}$ \\
\hline \multirow[t]{2}{*}{ VDH483 } & $11,5 \mathrm{Ab}$ & $8,5 \mathrm{Bc}$ & $8,1 \mathrm{~B}$ & $5,0 \mathrm{Cb}$ & $13,5 \mathrm{Ca}$ \\
\hline & \multicolumn{5}{|c|}{ N-NH3/NT } \\
\hline M742 & $6,0 \mathrm{Aab}$ & 7,9Aa & $9,1 \mathrm{Aa}$ & $7,8 \mathrm{Aa}$ & $6,6 \mathrm{Ab}$ \\
\hline MG4 (MO) & $6,1 \mathrm{Aab}$ & $7,8 \mathrm{Aa}$ & $7,1 \mathrm{Aa}$ & $6,3 \mathrm{Aa}$ & $8,9 \mathrm{Ab}$ \\
\hline PM92007 (MO) & $8,8 \mathrm{Ba}$ & $7,1 \mathrm{Ba}$ & $9,1 \mathrm{Ba}$ & $7,8 \mathrm{Ba}$ & $12,5 \mathrm{Aa}$ \\
\hline \multirow[t]{2}{*}{ VDH483 (MO) } & $6,4 \mathrm{Ab}$ & $6,7 \mathrm{Aa}$ & $7,3 \mathrm{Aa}$ & $6,1 \mathrm{Aa}$ & $8,5 \mathrm{Ab}$ \\
\hline & \multicolumn{5}{|c|}{$\mathrm{pH}$} \\
\hline M742 (SIL) & $4,2 \mathrm{Ca}$ & $4,0 \mathrm{Ca}$ & $4,0 \mathrm{Ca}$ & $4,5 \mathrm{Bb}$ & $4,8 \mathrm{Aab}$ \\
\hline MG4 (SIL) & $4,2 \mathrm{Ca}$ & $3,9 \mathrm{Da}$ & 3,9Dab & $4,6 \mathrm{Bab}$ & $5,0 \mathrm{Aa}$ \\
\hline PM92007 (SIL) & $4,1 \mathrm{Ba}$ & $3,8 \mathrm{Ca}$ & $3,8 \mathrm{Cb}$ & $4,0 \mathrm{Bc}$ & $5,0 \mathrm{Aa}$ \\
\hline VDH483 (SIL) & $4,1 \mathrm{Ba}$ & $4,0 \mathrm{Ba}$ & $4,0 \mathrm{Bab}$ & $4,7 \mathrm{Aa}$ & $4,7 \mathrm{Ab}$ \\
\hline
\end{tabular}

Letras maiúsculas distintas na mesma linha indicam diferenças $(\mathrm{P}<0,05)$ para cada híbrido nas diferentes idades de corte.

Letras minúsculas distintas na mesma coluna indicam diferenças $(\mathrm{P}<0,05)$ entre os híbridos (material original ou silagem) na mesma idade de corte.

Ac. acético, $\mathrm{CV}=17,4 \%$; ac. propiônco; $\mathrm{CV}=31,6 \%$; ac.buritíco, $\mathrm{CV}=127,6 \%$; $\mathrm{N}-\mathrm{NH}_{3} / \mathrm{NT}, \mathrm{CV}=18,1 \% ; \mathrm{pH}, \mathrm{CV}=18,1 \%$. Teste SNK $(\mathrm{P}<0,05)$.

Os teores de ácido lático diminuíram $(\mathrm{P}<0,05)$ com o avanço do estádio de maturação da planta e variaram de $31,5 \%$ para o genótipo MG4, na idade de corte de 90 dias, até $6,2 \%$ para o genótipo PM92007, na idade de corte de 118 dias. Segundo o critério proposto por Paiva (1976), para o ácido lático, todos os genótipos, em todas as idades de corte, produziram silagens de boa qualidade sob o aspecto de fermentação. Os adequados teores de ácido lático podem ser indicativos de que não houve limitação de substratos para a fermentação.

Os teores de nitrogênio amoniacal dos genótipos não diferiram $(\mathrm{P}>0,05)$ com o avanço do estádio de maturação, exceto para o genótipo PM92007, cujo aumento ocorreu aos 118 dias de idade ao corte. De acordo com critério proposto por Benachio (1965), citado por Borges (1995), somente a silagem do cultivar PM92007, na idade de corte de 118 dias, pode ser classificada como boa, sendo as outras silagens de todos os outros genótipos classificadas como muito boas. Isso indica que a proteólise ocorrida nos silos, durante o processo de ensilagem, foi pequena.

Com relação aos valores de $\mathrm{pH}$, segundo a avaliação proposta por Paiva (1976), nas idades 97 e 104 dias, as silagens são classificadas como de boa qualidade. Para os genótipos PM92007 e VDH483, na idade de 90 dias, as silagens também podem ser classificadas como de boa 
qualidade. Para o genótipo PM92007, as silagens podem ser classificadas como de boa qualidade até a idade de 111 dias e as dos demais genótipos, na idade de corte de 111 dias. Todos os genótipos, na idade de corte de 118 dias, seriam classificados como de qualidade ruim.

\section{CONCLUSÕES}

Todos os cultivares apresentaram potencial para serem utilizados para produção de silagens. O melhor momento de colheita, tendo em vista as variáveis de qualidade de silagens para os genótipos M742, MG4, VDH483 foi obtido aos 104 dias de idade de corte, enquanto, para o genótipo PM92007, foi aos 111 dias de idade.

\section{REFERÊNCIAS BIBLIOGRÁFICAS}

BAILEY, R.W. Quantitative studies of ruminant digestion. II. Loss of ingested plant carbohydrates from teh reticulo rúmen. N. Z. J. Agric. Res., v.10, p.15-32, 1967.

BORGES, A.L.C.C. Qualidade de silagens de híbridos de sorgo de porte alto, com diferentes teores de tanino e umidade no colmo, e seus padrões de fermentação. 1995. 87f. Dissertação (Mestrado) - Escola de Veterinária, Universidade Federal de Minas Gerais, Belo Horizonte.

CHURCH, D.C. The ruminant animal digestive physiology and nutrition. New Jersey: Prentice Hall, 1988. 564p.

GONÇALVES, L.C.; TOMICH, T.T. Utilização do girassol como silagem para alimentação bovina. In: SIMPÓSIO NACIONAL SOBRE A CULTURA DO GIRASSOL, 1., 1999, Itumbiara. Anais... Londrina: EMBRAPA, 1999. p.21-30.

JAMES, C.S. Analytical chemistry of foods. London: Blackie Academic Professional, 1995. p.37-67.

McDONALD, P.; HENDERSON, A.R.; HERON, S. The biochemistry of silage. 2.ed. Marlow: Chalcombe Publications, 1991. 340p.
OFFICIAL methods of analysis. 16.ed. Washington, DC: AOAC International, 1995.

OFFICIAL methods of analysis. 13.ed. Washington, DC: AOAC International, 1980. $1015 \mathrm{p}$.

PAIVA, J.A.J. Qualidade da silagem da região metalúrgica de Minas Gerais. 1976. 85f. Dissertação (Mestrado). Escola de Veterinária, Universidade Federal de Minas Gerais, Belo Horizonte.

RODRIGUEZ, N.M.; GONÇALVES, L.C.; NOGUERA, F.A.S. et al. Silagem de sorgo de porte baixo com diferentes teores de tanino e de umidade no colmo. I- $\mathrm{pH}$ e teores de matéria seca e de ácidos graxos durante a fermentação. Arq. Bras. Med. Vet. Zootec., v.51, p.485-490, 1999.

SISTEMA de análises estatísticas e genéticas SAEG. Viçosa: UFV, 1997. 52p.

STEHLING, C.A.V. Avaliação da qualidade das silagens de quatro cultivares de girassol contendo aditivos. 2001. 64f. Dissertação (Mestrado) - Escola de Veterinária, Universidade Federal de Minas Gerais, Belo Horizonte.

TILLEY, J.M.A.; TERRY, R.A. Two stage technique for the "in vitro"digestion of forage crops. J. Br. Grass Soc., v.18, p.104-111, 1963.

TOMICH, T.R. Avaliação do potencial forrageiro e das silagens de treze cultivares de girassol. 1999. 81f. Dissertação (Mestrado) Escola de Veterinária, Universidade Federal de Minas Gerais, Belo Horizonte.

VAN SOEST, P.J. Nutritional ecology of the ruminant. 2.ed. Ithaca: Cornell University, 1994. 476p.

ZAGO, C.P.; POZAR, G. Época de corte de sorgo (Sorghum bicolor L. Moench) e sua influência sobre a porcentagem de matéria seca e de panícula. In: REUNIÃO ANUAL DA SOCIEDADE BRASILEIRA DE ZOOTECNIA, 28., 1991, João Pessoa. Anais... João Pessoa:SBZ, 1991. p.61. 